\title{
Alinhamento rotacional do componente femoral na artroplastia total do joelho*
}

\section{Femoral rotational alignment in total knee arthroplasty}

\author{
Lúcio Honório de Carvalho JúnioR ${ }^{1}$, Matheus Braga Jacoues Gonçalves², \\ Luiz Fernando Machado Soares ${ }^{2}$, Rafael Cavalcanti Silva ${ }^{3}$
}

\section{RESUMO}

Objetivo: Avaliar a eficiência dos guias de orientação rotacional utilizados na realização da artroplastia total do joelho. Avaliar a possível correlação entre o eixo anatômico pré-operatório e a capacidade do guia em posicionar corretamente o componente. Métodos: Foram avaliadas 64 artroplastias confrontando a orientação sugerida pelo guia com aquela mostrada pela linha de Whiteside. Os casos de divergência foram registrados e confrontados com o eixo anatômico pré-operatório. Resultado: Não houve divergências entre o alinhamento rotacional medido pelos dois métodos nos joelhos alinhados e naqueles com alinhamento em varo. Foram encontradas divergências entre o alinhamento rotacional sugerido pelo guia e aquele mostrado pela linha de Whiteside em sete dos 64 joelhos $(10,93 \%)$. Em todos os pacientes com discrepância, o alinhamento préoperatório era valgo. Conclusão: Os guias de orientação rotacional do componente femoral na artroplastia total do

* Trabalho realizado no Hospital Madre Teresa de Belo Horizonte (MG), Brasil.

1. Doutor, Professor Adjunto do Departamento do Aparelho Locomotor da Universidade Federal de Minas Gerais - UFMG - Belo Horizonte (MG), Brasil; Membro do Grupo do Joelho do Hospital Madre Teresa - Belo Horizonte (MG), Brasil.

2. Membro do Grupo do Joelho do Hospital Madre Teresa - Belo Horizonte (MG), Brasil.

3. Especializando em Cirurgia do Joelho do Hospital Madre Teresa - Belo Horizonte (MG), Brasil.

Endereço para correspondência: Rua Olavo Carsalade Vilela, 264, Residencial Ipê da Serra -34000-000 - Nova Lima (MG), Brasil.

E-mail: luciohcj@medicina.ufmg.br

Recebido em 13/3/07. Aprovado para publicação em 14/8/07.

Copyright RBO2007 joelho mostraram-se coincidentes com a orientação obtida pela linha de Whiteside nos joelhos com eixo anatômico em varo e naqueles alinhados. Nos joelhos com eixo anatômico em valgo, a referência dos guias não se mostrou coincidente com aquela sugerida pela mesma linha.

Descritores - Artroplastia do joelho/métodos; Prótese do joelho; Rotação; Estudos de avaliação

\section{ABSTRACT}

Objective: To evaluate the efficiency of rotational wire guides used to perform total knee arthroplasty. To evaluate the possible correlation between the preoperative anatomic axis and the capacity of the wire guide to properly position the component. Methods: 64 arthroplasties were evaluated by comparing the position suggested by the wire guide to the position shown by the Whiteside line. Discrepant cases were registered and compared to the preoperative anatomic axis. Result: No discrepancies were found between the rotational alignment measured by the two methods in aligned knees and knees with varus alignment. Discrepancies were found between the rotational alignment suggested by the wire guide and the alignment shown by the Whiteside line in seven of the 64 knees (10.93\%). In all patients that showed discrepancy, the preoperative alignment was valgus. Conclusion: Rotational wire guides of the femoral component in total knee arthroplasty do coincide with the position achieved by the Whiteside line in knees whose anatomic axis is varus and in aligned knees. In valgus knees, the reference of the wire guides did not coincide with the position suggested by the said line.

Keywords - Arthroplasty, replacement, knee/methods; Knee prosthesis; Rotation; Evaluation studies 


\section{INTRODUÇÃO}

$\mathrm{O}$ alinhamento rotacional inadequado do componente femoral na artroplastia total do joelho afeta a estabilidade mediolateral em flexão e o trajeto da patela no seu encaixe sobre a tróclea ${ }^{(1-3)}$.

Vários métodos têm sido propostos para determinar o correto alinhamento rotacional desse componente. A linha troclear ântero-posterior (linha de Whiteside) e o eixo transepicondilar são dois exemplos ${ }^{(4-5)}$.

Alguns instrumentais de diversos tipos de próteses têm utilizado a rotação externa de $3^{\circ}$ a partir de uma referência nos côndilos posteriores como elemento para atingir o correto posicionamento rotacional do componente femoral.

O objetivo deste trabalho é avaliar a eficiência desse tipo de instrumental na identificação do correto posicionamento rotacional usando como parâmetro a linha de Whiteside. Será avaliada a possível correlação entre o eixo anatômico pré-operatório e a capacidade do guia em posicionar corretamente o componente.

\section{MÉTODOS}

Entre janeiro de 2006 e janeiro de 2007, foram operados pelos autores no Hospital Madre Teresa de Belo Horizonte, MG, 63 pacientes submetidos à artroplastia total do joelho com três diferentes tipos de próteses, todas elas usando guias rotacionais que se apoiavam nos côndilos posteriores.

Foram operados 64 joelhos de 63 pacientes, sendo 13 do sexo masculino e 50 do feminino, com idade variando entre 57 e 82 anos, com média de 71,8 anos. Todos os pacientes, com uma única exceção, eram portadores de osteoartrose primária. O alinhamento pré-operatório (medido pelo eixo anatômico) encontrou 10 joelhos com alinhamento em valgo (variando entre $1^{\circ}$ e $20^{\circ}$ ), cinco com o membro alinhado e 49 com alinhamento em varo (variando entre $2^{\circ}$ e $20^{\circ}$ ).

Das 64 próteses realizadas (em um caso a artroplastia foi bilateral), 18 eram próteses Nex Gen ${ }^{\circledR}$ produzidas pela Zimmer Corporation (EUA), 15 eram próteses Duracon $^{\circledR}$ e 31 eram próteses $S$ corpio ${ }^{\circledR}$, ambas produzidas pela Stryker Corporation (EUA).

Durante a cirurgia, antes da confecção do corte do fêmur distal era marcada a linha de Whiteside, conforme mostrado na figura 1. Após o corte na extremidade distal do fêmur e usando como referência os orifícios de marcação do próprio instrumental, era medida com goniômetro plástico a divergência em graus entre as duas marcações.

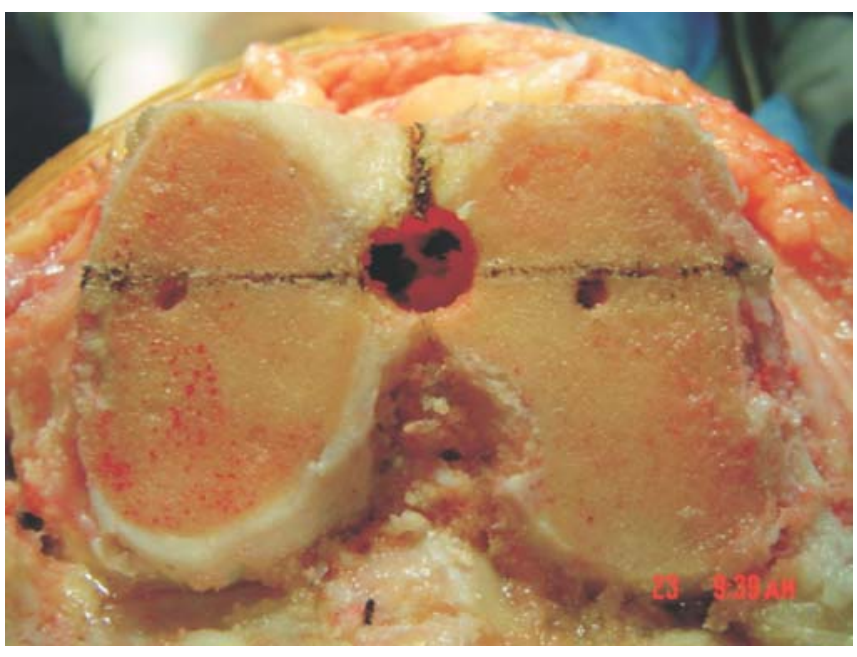

Figura 1 - Fotografia peroperatória apresentando a linha de Whiteside e o eixo sugerido por essa e pelo guia de orientação rotacional da prótese, mostrando coincidência entre eles

Foram realizadas radiografias panorâmicas de membros inferiores em ântero-posterior ortostático de todos os pacientes para medida do eixo anatômico do joelho.

Para análise dos dados foi comparada a possível relação entre o alinhamento anatômico no plano frontal e a diferença entre as duas medidas de orientação rotacional. Foram comparadas, ainda, as possíveis divergências em relação aos três tipos de próteses utilizadas.

Como se trata de investigação em parâmetros normalmente levantados no pré e peroperatórios desse tipo de cirurgia e por orientação da Comissão de Ética em Pesquisa da Instituição, não foi solicitada aprovação formal da pesquisa naquela comissão. Apesar disso, foram obtidos consentimentos pós-informados por escrito de todos os pacientes autorizando a utilização de seus dados na pesquisa.

A análise estatística dos dados foi feita avaliando-se a dispersão e as medidas de tendência central, usando-se como nível de significância o valor $p<0,05$.

\section{RESULTADOS}

Foram encontradas divergências entre o alinhamento rotacional sugerido pelo guia e aquele mostrado pela linha de Whiteside em sete dos 64 joelhos (10,93\%), com diferenças variando entre $2^{\circ}$ e $5^{\circ}$ de rotação interna. Em todos os pacientes com discrepância, o alinhamento pré-operatório era valgo, variando entre $3^{\circ}$ e $20^{\circ}$.

Não houve divergências entre o alinhamento rotacional medido pelos dois métodos nos joelhos alinhados e naqueles com alinhamento em varo. 
Dos sete pacientes com divergência entre as medidas, dois $(11,1 \%)$ usaram o guia da prótese Nex Gen ${ }^{\circledR}$; um $(6,7 \%)$, da prótese Duracon $^{\oplus}$; e quatro $(12,9 \%)$, da prótese Scorpio $^{\circledR}$. A distribuição entre os três grupos não mostrou diferenças entre as próteses: $p>0,05$.

\section{DISCUSSÃO}

O alinhamento rotacional femoral afeta a excursão patelar, os pontos de contato e as pressões femoropatelares ${ }^{(3-7)}$, porém pouca informação existe a respeito da sua relação com o componente patelar ${ }^{(8)}$. Pacientes com rotação externa dos componentes entre 0 e $10^{\circ}$ não apresentaram complicações femoropatelares. Anouchi et al, em estudo em cadáveres, e Whiteside et al, em pacientes, mostraram que o alinhamento do componente femoral em rotação interna, além das complicações patelares, pode causar ainda instabilidade do joelho em flexão $0^{3-}$ 4). Essa instabilidade ocorre pela diferença de tamanho entre as brechas em extensão e flexão causada pela alteração das dimensões relativas dos côndilos posteriores em flexão. Clinicamente, essa inadequação pode manifestar-se como dor metafisária tibial medial, dor envolvendo os tendões da pata de ganso, sensação de instabilidade e derrame articular recorrente $^{(6)}$.

O alinhamento rotacional incorreto do componente femoral diminuiu com a melhora no desenho dos componentes, na técnica e no instrumental cirúrgico, mas ainda continua sendo a causa mais comum de cirurgias de revisão em função das suas consequiências sobre o correto funcionamento da prótese total do joelho ${ }^{(6-7)}$.

Vários métodos foram propostos para estabelecer o correto alinhamento rotacional do componente femoral, sendo o eixo transepicondilar ${ }^{(5)}$ e a linha de Whiteside ${ }^{(4)}$ as mais citadas na literatura. Alguns autores divergem quanto ao método de maior acurácia e reprodutibilidade. Berger et al e Poilvache et al consideram ser o eixo transepicondilar mais fácil de localizar e mais consistente ${ }^{(5,7)}$. Para eles, é muitas vezes difícil de definir a linha de Whiteside pela presença de desgaste troclear ou de osteófitos intercondilares. Nos casos com displasia grave da tróclea, os mesmos autores apontam para a possível indução a rotação externa excessiva do componente femoral quando a linha de Whiteside é utilizada, além do fato de ela não poder ser facilmente definida nos casos de revisão, em que o eixo transepicondilar é mais confiável como referência de orientação rotacional.

Com opinião oposta, Arima et al e Whiteside et al demonstraram a dificuldade em localizar o eixo transepicondilar durante a cirurgia devido à cobertura de partes moles sobre os epicôndilos ${ }^{(1,4)}$. Consideram essa dificuldade causa de erros rotacionais grosseiros e sugerem a linha troclear ântero-posterior como o método mais eficiente de estabelecer o alinhamento rotacional.

As medidas realizadas durante a cirurgia utilizando goniômetro plástico podem comprometer em parte a precisão dos achados desse estudo, pois, sem dúvida, são menos precisas que aquelas realizadas por outros métodos como a tomografia, fato já salientado por Poilvache $e t a^{(7)}$.

Os resultados desse estudo mostraram coincidência entre a orientação rotacional obtida com os guias de referência condilar posterior e aquela conseguida com a linha de Whiteside nos joelhos com eixo anatômico alinhado ou em varo. Nos joelhos com eixo anatômico em valgo, a orientação obtida com os guias não coincidiu com aquela sugerida pela linha de Whiteside. Whiteside et al e Siston et al encontraram resultados semelhantes ${ }^{(4,9)}$.

Nos joelhos em varo ou alinhados, o formato e o tamanho dos côndilos femorais medial e lateral, na sua parte posterior, são muito parecidos. Não há hipoplasia posterior do côndilo medial, mesmo nos casos com varo muito expressivo ${ }^{(10)}$. A referência posterior, entretanto, fica comprometida nos joelhos com valgo acentuado, uma vez que, nestes, observa-se importante distorção da arquitetura articular femoral com hipoplasia anterior, distal e posterior do côndilo lateral, tornando a obtenção do alinhamento rotacional especialmente difícil $^{(1,4)}$. Tal dificuldade provoca rotação interna indesejada do componente femoral.

É possível que os eixos definidos nesse estudo estejam em rotação externa em relação ao eixo transepicondilar verdadeiro, fato já observado em outros estudos pela tendência dos cirurgiões em errar no sentido de aumentar a rotação externa, procedimento conhecido e utilizado para evitar os resultados indesejáveis relacionados à rotação interna ${ }^{(3,9)}$. Entretanto, a rotação externa excessiva também pode trazer problemas, como aumento das forças de cisalhamento no componente patelar e instabilidade em flexão devido ao aumento da brecha medial em flexão $0^{(9)}$.

O alinhamento rotacional do componente femoral pode também ser definido no pré-operatório, marcando-se o eixo transepicondilar e o eixo condilar posterior através de exames de imagem. As referências ósseas para a marcação não são sempre visíveis em imagens unidimensionais. Para Tan et al, as referências ósseas são melhor identificadas em imagens seriadas de ressonância magnética ou tomografia computadorizada (TC) em três dimensões ${ }^{(11)}$. Apesar do custo, a utilização da TC se justifica para avaliação de casos com suspeita de mau 
posicionamento, uma vez que é a única técnica estabelecida, validada e reprodutível para medir a rotação dos componentes da prótese total do joelho ${ }^{(5-6,12)}$.

O uso de sistemas de navegação tende a aumentar a precisão do correto posicionamento dos componentes e pode ajudar a evitar erros, não apenas no alinhamento frontal, mas também no rotacional. Contudo, para Siston et al, o uso da navegação não melhorou os resultados do posicionamento rotacional do componente femoral e, assim como outros métodos, necessita refinamento ${ }^{(9)}$. Em seu estudo, demonstraram não existir diferenças na média de erros associados com diversos métodos manuais e assistidos por computador para orientação do alinhamento rotacional, além de grande varia-

\section{REFERÊNCIAS}

1. Arima J, Whiteside LA, McCarthy DS, White SE. Femoral rotational alignment based on the anteroposterior axis, in total knee arthroplasty in a valgus knee. A technical note. J Bone Joint Surg Am. 1995;77(9):1331-4.

2. Akagi M, Matsusue Y, Mata T, Asada Y, Horiguchi M, Iida H, Nakamura T. Effect of rotational alignment on patellar tracking in total knee arthroplasty. Clin Orthop Relat Res. 1999;(366):155-63.

3. Anouchi YS, Whiteside LA, Kaiser AD, Milliano MT. The effects of axial rotational alignment of the femoral component on knee stability and patellar tracking in total knee arthroplasty demonstrated on autopsy specimens. Clin Orthop Relat Res. 1993;(287):170-7.

4. Whiteside LA, Arima J. The anteroposterior axis for femoral rotational alignment in valgus total knee arthroplasty. Clin Orthop Relat Res. 1995; (321):168-72.

5. Berger RA, Rubash HE, Seel MJ, Thompson WH, Crossett LS Determining the rotational alignment of the femoral component in total knee arthroplasty using the epicondilar axis. Clin Orthop Relat Res. 1993; (286):40-7.

6. Stöckl B, Nogler M, Rosiek R, Fischer M, Krismer M, Kessier O Navigation improves accuracy of rotational alignment in total knee arthroplasty. Clin Orthop Relat Res. 2004;(426):180-6. ção entre eles quando comparados com o eixo transepicondilar localizado após a remoção de partes moles em cadáveres. Consideram a habilidade individual de cada cirurgião como o fator mais importante no correto posicionamento rotacional, independente da referência escolhida ${ }^{(9)}$.

\section{CONCLUSÃO}

Os guias de orientação rotacional do componente femoral na artroplastia total do joelho mostraram-se coincidentes com a orientação obtida pela linha de Whiteside nos joelhos com eixo anatômico em varo e naqueles alinhados. Nos joelhos com eixo anatômico em valgo, a referência dos guias não se mostrou coincidente com aquela sugerida pela mesma linha.

7. Poilvache PL, Insall JN, Scuderi GR, Font-Rodriguez DE. Rotational landmarks and sizing of the distal femur in total knee arthroplasty. Clin Orthop Relat Res. 1996;(331):35-46.

8. Berger RA, Crossett LS, Jacobs JJ, Rubash HE. Malrotation causing patellofemoral complications after total knee arthroplasty. Clin Orthop Relat Res. 1998;(356):144-53.

9. Siston RA, Patel JJ, Goodman SB, Delp SL, Giori NJ. The variability of femoral rotational alignment in total knee arthroplasty. J Bone Joint Surg Am. 2005;87(10):2276-80.

10. Matsuda S, Matsuda H, Miyagi T, Sasaki K, Iwamoto Y, Miura H. Femoral condyle geometry in the normal and varus knee. Clin Orthop Relat Res. 1998;(349):183-8.

11. Tan CM, Liau JJ, Chen WT, Cheng CK. The Accuracy of Posterior Condylar Angles Measured by One MR Image. Clin Orthop Relat Res. 2006; [Epub ahead of print]

12. Cui WQ, Won YY, Baek MH, Kim KK, Cho JH. Variations of the 'granpiano sign' during total knee replacement. A computer-simulation study. J Bone Joint Surg Br. 2006;88(11):1441-7. 